\title{
BMJ Open Is change in mental distress among adolescents predicted by sedentary behaviour or screen time? Results from the longitudinal population study The Tromsø Study: Fit Futures
}

Ida Marie Opdal (D , ${ }^{1}$ Bente Morseth, ${ }^{2}$ Bjørn-Helge Handegård, ${ }^{3}$ Kjersti R Lillevoll, ${ }^{1}$ Wendy Nilsen, ${ }^{4}$ Christopher Nielsen, ${ }^{5}$ Anne-Sofie Furberg, ${ }^{6,7}$ Simon Rosenbaum, ${ }^{8,9}$ Kamilla Rognmo ${ }^{1}$

To cite: Opdal IM, Morseth B, Handegård B-H, et al. Is change in mental distress among adolescents predicted by sedentary behaviour or screen time? Results from the longitudinal population study The Tromsø Study: Fit Futures. BMJ Open 2020;10:e35549. doi:10.1136/ bmjopen-2019-035549

- Prepublication history for this paper is available online To view these files, please visit the journal online (http://dx.doi. org/10.1136/bmjopen-2019035549).

Received 05 November 2019 Revised 10 January 2020 Accepted 13 January 2020
Check for updates

(C) Author(s) (or their employer(s)) 2020. Re-use permitted under CC BY-NC. No commercial re-use. See rights and permissions. Published by BMJ.

For numbered affiliations see end of article.

Correspondence to Ida Marie Opdal; ida.m.opdal@uit.no

\section{ABSTRACT}

Objective There is growing interest in the relationship between sedentary behaviour and mental distress among adolescents, but the majority of studies to date have relied on self-reported measures with poor validity. Consequently, current knowledge may be affected by various biases. The aim of this study was to investigate the cross-sectional and longitudinal association between (1) objectively measured sedentary time and (2) self-reported screen time with mental distress among adolescents participating in The Troms $\emptyset$ Study: Fit Futures, in order to see if the association is dependent on mode of measurement of sedentary behaviour.

Design Prospective study.

Setting Sample drawn from upper secondary school students (mean age 16.3 years at baseline) from two municipalities in Northern Norway participating in The Tromsø Study: Fit Futures 1 and 2.

Participants 686 adolescents (54.5\% female), with complete self-reported and accelerometer data after multiple imputation.

Primary outcome measures Mental distress assessed via the Hopkins Symptom Checklist-10 (HSCL-10). Results Minutes in sedentary behaviour measured by accelerometer showed no significant relationship with mental distress in neither crude, partly adjusted nor multiple adjusted hierarchic linear regression analyses. Self-reported screen time was positively associated with mental distress in all analyses (multiple adjusted, $\mathrm{B}=0.038, \mathrm{p}=0.008,95 \% \mathrm{Cl} 0.010$ to 0.066 ). However, the effect was small.

Conclusions Self-reported screen time was associated with slightly elevated mental distress 2 years later, whereas objectively measured minutes in sedentary behaviour was not, indicating a discrepancy in the results depending on measurement methods.

Mental disorders commonly arise during the teenage years, ${ }^{1}$ signifying that adolescence may be a crucial period for preventing the development of mental health problems.
Strengths and limitations of this study

- Use of both objective measure of sedentary behaviour and self-reported screen time.

- A prospective study design across 2 years in middle and late adolescence.

- Analysis controlled for several relevant covariates, for example, socioeconomic status, social network and physical activity levels.

- No collected self-reported data on total volume and different types of sedentary behaviour other than screen time.

- Sample contains students from two municipalities in, restricting the generalisability of the study.

The importance of prevention is increasingly emphasised as more than 1 in 10 children and adolescents suffer from a mental disorder. ${ }^{2}$ Interestingly, the onset of symptoms of depression and anxiety during the adolescent period coincides with a decrease in physical activity that also occurs during this period. $^{3}$

Physical activity is thought to play a significant role in protecting against poor mental health in adolescents, ${ }^{5}$ but also in children, ${ }^{67}{ }^{7}$ adults $^{8}$ and the elderly. ${ }^{9}$ The effect of physical activity is theoretically explained by positive physiological responses, such as higher levels of norepinephrine, endorphins and serotonin, and lower hormonal responses from stress, ${ }^{10}{ }^{11}$ but could also be explained by psychological responses, such as feelings of mastery and control, higher self-esteem, and distracting from stressors, negative thoughts and rumination. ${ }^{101213}$ However, a review done by Bailey et $a l^{14}$ concluded that even though there are promising results indicating that physical activity may have a treatment effect 
on mental distress, there are methodological weaknesses that obstruct a clear understanding of the association. In addition, other studies have not found an association between physical activity and mental health. ${ }^{15-17}$

If there are physiological and psychological benefits of physical activity, this could indicate that the opposite of engaging in physical activity-sedentary behaviours that involve sitting or resting-could potentially increase symptoms of mental distress. However, high levels of sedentary time do not necessarily correlate with low levels of physical activity, and as such, physical activity and sedentary behaviours are not necessarily mutually exclusive. ${ }^{18}$ Because a person may be both physically active and sedentary over a period, adjusting for physical activity is essential when investigating the degree to which sedentary behaviour is an independent risk factor of mental health problems among adolescents.

Until recently, research on the association between sedentary behaviour and mental health has been restricted to using self-reported measurements of sedentary behaviour. The majority of the existing studies on the association between sedentary behaviour and mental health have operationalised the term sedentary behaviour as hours of self-reported screen time. ${ }^{19}$ In general, previous studies based on self-report conclude that there is a moderate positive association between screen time and depressive symptoms. ${ }^{19-24} \mathrm{~A}$ recent study investigating the effects of screen time on adolescent well-being (operationalised as high levels of well-being and low levels of mental health problems) found a significant negative association. ${ }^{25}$ However, screen time only accounted for $0.4 \%$ of the variance in broadly defined well-being, suggesting that screen time is associated with reductions in well-being only to a very small extent. A recent Norwegian study from 2019 also reported that screen time is associated with slight increases in depression, behaviour problems and alcohol use among adolescents. ${ }^{26}$

Despite screen time being the most common measure of sedentary behaviour, the validity of the measure is questionable. Several studies have shown that screen time is a poor estimate of sedentary behaviour compared with objective measures. ${ }^{27}$ This means that the evidence base regarding the relationship between sedentary behaviour and mental distress rests on an unreliable foundation. Hamer $e t a l^{28}$ investigated the association between objectively assessed and self-reported sedentary time with mental health in adults, and found that the highest tertile of objectively assessed sedentary behaviour was associated with adverse mental health, but that the context-specific sedentary time, TV viewing, was far less consistently associated. This may indicate that the statistical relationship between sedentary behaviour and mental health may depend on measurement issues, and having both objectively measured and self-report data on sedentary behaviours may provide important insight regarding the association.

The current study aimed to investigate the crosssectional and longitudinal association between (1) objectively measured sedentary time and (2) self-reported screen time with mental distress among adolescents participating in The Tromsø Study: Fit Futures, in order to see if the association is dependent on mode of measurement of sedentary behaviour. Objectively measured minutes in moderate to vigorous physical activity (MVPA) will serve as a covariate in the analyses, alongside demographic, psychosocial and health-related covariates, to investigate to which the degree sedentary behaviour is an independent risk factor of mental distress among adolescents. In the longitudinal analyses, baseline levels of mental distress will be adjusted for, enabling the coefficients of the analyses to be interpreted as indicators of change in the outcome between baseline and 2 years later.

\section{METHOD}

\section{Study design and sample}

The present study is based on data from a longitudinal population-based general health study, The Troms $\varnothing$ Study: Fit Futures, conducted in two waves, 2 years apart. In the first wave (T1, baseline) in 2010-2011, all first-level upper secondary school students in two municipalities were invited to participate. In total, 1117 students were invited, and 1038 (92.9\%) participated. The second wave (T2, follow-up) was conducted in 2012-2013, where all T1 attendees and all students at the third level of the same upper secondary schools as in T1 were invited to participate. In total, 1129 students were invited, out of which $870(77 \%)$ participated. Of these, 694 (67\%) students also participated at $\mathrm{T} 1$. The majority of the participating sample was 15-17 years old at T1 (middle adolescence) and 17-19 years old at T2 (late adolescence). The study is described in detail elsewhere..$^{29} 30$

The Fit Futures study included a web-based questionnaire, clinical examinations and interviews performed by trained research nurses at the study site. All participants aged 16 or older provided informed consent, as stated in $\$ 17$ in the Norwegian Health Research Act; for participants younger than age 16 , consent was also given by a guardian.

\section{Patient and public involvement \\ No patients involved.}

\section{Measurements}

Sedentary time and physical activity

Percentage of sedentary behaviour per valid day and minutes in MVPA were measured using the ActiGraph GT3X accelerometer at T1. The participants were instructed to wear the ActiGraph on their dominant hip for 8 days, and to only take it off when sleeping, showering or swimming.

The ActiGraph measures acceleration in movement, referred to as 'counts'. The raw accelerometer files was reduced to $10 \mathrm{~s}$ epochs using the ActiLife software owned by the manufacturer, ${ }^{31}$ the $10 \mathrm{~s}$ epochs was further summed to $1 \mathrm{~min}$. Due to the possible variation in physical 
activity during the period of measurement, participants with at least 10-hour wear time for at least 4 out of 7 days were considered to have valid data. The activity variables, minutes in sedentary behaviour per valid day and minutes in MVPA per valid day for each participant, were based on the cut-points by Freedson et al, ${ }^{32}$ sedentary behavior $<100$ counts per minute and MVPA $>1952$ counts per minute. The percentage of the day spent in sedentary behaviour was used as the predictor variable, and minutes in MVPA per valid day contributed as a covariate.

Estimated hours spent per day watching a screen (PC, TV and DVD) outside of school time for weekdays and weekends was reported in the general questionnaire ("How many hours per day do you spend by the PC, watching TV, DVD etc outside school during weekdays/weekends?"). The response categories ranged from 'none' to 'about 10 hours or more'. These ordinal variables were recoded in order to approximate a continuous variable and thus facilitate a more appropriate interpretation in the regression analyses ('none' was changed into 0 , 'about 0.5 hours' into 0.5 , 'about 1 to 1.5 hours' into 1.25 , 'about 2 to 3 hours' into 2.5, 'about 4 to 6 hours' into 5 , 'about 7 to 9 hours' into 8 , and 'about 10 or more hours' into 10).

\section{Mental distress}

The Hopkins Symptom Checklist-10 (HSCL-10) was included in the general questionnaire at $\mathrm{T} 1$ and $\mathrm{T} 2$, and measures symptoms of anxiety (four items) and depression (six items) during the previous 7 days. The internal and external validity and reliability of the HSCL-10 has been found acceptable in a number of studies (eg, previous works ${ }^{33}{ }^{34}$ ), and correlates well with depression diagnosed using diagnostic interview. ${ }^{35}$ Response categories were 'none' (1), 'slightly' (2), 'much' (3) and 'very much' (4). The average for the scale was then calculated and further standardised, and the standardised score at T2 served as the outcome variable in the analyses. The analysis controlled for the average score at $\mathrm{T} 1$.

\section{Covariates}

Several variables may have an impact on sedentary behaviour and mental distress, and thus, we have included demographic variables (eg, socioeconomic status, ${ }^{36}$ sex and age ${ }^{37}$ ), health variables (eg, smoking, ${ }^{38}$ chronic pain, ${ }^{39}$ body mass index (BMI ${ }^{40}$ and sleep ${ }^{41}$ ) and social variables (eg, social network ${ }^{42}$ ) as covariates in the analyses.

\section{Demography at $\mathrm{T} 1$}

Sex was included in the analyses as a possible confounder. Further, the respondents were asked to indicate the highest level of completed education of their mother and father, which served as a proxy for socioeconomic status (high/low, with high education meaning higher than secondary vocational education) in the analyses. As the season of the year might significantly affect the level of physical activity, a seasonal variable indicating the time of
ActiGraph measurement at T1 and T2 (summer: May to June and September to October, or winter: November to April) was included in the analyses as a covariate.

\section{Health and psychosocial variables at $\mathrm{T} 1$}

The respondents reported if they had persistent pain that had lasted 3 months or longer (yes/no), and rated their overall perception of their own health, measured by a five-point Likert type scale. A variable on social network was made by a mean score of five items asking about the degree to which the respondents find it hard to make friends, have many friends, feel accepted among his/her peers, feel liked among peers, and feel popular among peers. Sleep delay was measured by asking the respondents to indicate how long they normally lie awake before falling asleep (sleep onset latency) on weekdays and weekends. Response categories ranged from ' 30 min or less' to ' 3 hours or more'. A sleep onset latency over $30 \mathrm{~min}$ on more than 3 days per week is considered a clinical marker for insomnia. ${ }^{43}$ A dichotomous variable was created, with a cut-off at spending more than $30 \mathrm{~min}$ falling asleep $(0=$ sleep onset latency $30 \mathrm{~min}$ or less, 1=sleep onset latency over $30 \mathrm{~min}$ ). Weight and height were measured at the examination site and BMI was calculated by dividing the participants weight in $\mathrm{kg}$ by their height in metres squared.

\section{Treatment of missing data}

Multiple imputation (IBM SPSS V.25 for windows) was used to impute T1 ActiGraph data and socioeconomic status of participants who had questionnaire data from both T1 and 2 years later at T2. These variables represented a high proportion of the missing data and was imputed in order to maintain power and reduce possible bias due to missing values. Of the participants who had data on both T1 and T2, 464 (67\%) had valid ActiGraph data at T1, $505(71 \%)$ had valid data on socioeconomic status based on fathers' education and 529 (74\%) had valid data on socioeconomic status based on mothers' education. A predictive model consisting of all variables included in the analyses was used to create 50 imputed datasets that subsequently were pooled and analysed. After imputations, $35 \%$ of the data on percentage of sedentary behaviour per valid day, 35\% of MVPA, $26 \%$ of socioeconomic status of mother and $29 \%$ of socioeconomic status of father were imputed. After imputation, 686 participants had complete data on all the variables used in the analyses.

\section{Data analysis}

Descriptive statistics and Pearson product-moment correlation analysis between percentage of sedentary behaviour per valid day measured by accelerometer and self-reported screen time, in addition to the hierarchical linear regression analysis, were conducted using the statistical software IBM SPSS V.25. ${ }^{44}$ In order to investigate the research questions of interest, two sets of hierarchical linear regression analyses were conducted, analysing the 
degree to which percentage of sedentary behaviour (T1) and self-reported screen time (T1) are cross-sectionally (at $\mathrm{T} 1 /$ baseline) or longitudinally (change between $\mathrm{T} 1$ and T2) associated with mental distress. In the crosssectional analyses, percentage of sedentary behaviour or self-reported screen time was entered alone in the first model. In model 2, the demographic variables (sex, age, socioeconomic status and season) were entered, whereas health and psychosocial variables (BMI, sleep onset latency, self-reported health, social network, chronic pain and either percentage of sedentary behaviour or screen time (depending on which serves as the predictor variable in the analysis in question)) were entered as covariates in model 3. The same procedure was followed for the longitudinal analyses; however, in model 1, T1 symptoms of anxiety and depression were included in order to investigate residualised change in mental distress in relation to $\mathrm{T} 1$ percentage of sedentary behaviour and screen time. Interaction effects between percentage of sedentary behaviour and self-reported screen time were investigated in the multiple adjusted models to see if the relationship between percentage of sedentary behaviour and mental distress was dependent on levels of screen time, and vice versa, under the assumption that sedentary behaviour and screen time relate to different constructs. Interaction effects between sedentary behaviour/screen time and sex, mental health at T1, MVPA, social network and BMI was also investigated. In addition, the analyses were also conducted on complete cases only.

\section{RESULTS}

\section{Descriptive statistics}

Descriptive statistics are shown in table $1.86 .6 \%$ of the participants at T1 engaged in less MVPA than the recommended $60 \mathrm{~min}$ per day. ${ }^{45}$ On average, the adolescents reported 4 hours of screen time per day and were sedentary for more than 9 hours per day. Almost $80 \%$ of the accelerometer measurements occurred during wintertime. $20.1 \%$ of the participants at T1 had a HSCL-10 score over the cut-off of 1.85, identified as a threshold for indicating the presence of an internalising mental disorder among adolescents. ${ }^{34}$ At T2, $26.7 \%$ had a score over the cut-off. Finally, a majority of the participants reported that their mother and father had a low level of education (see table 1).

The correlation between percentage of sedentary behaviour per valid day measured by accelerometer and self-reported screen time was $0.20, \mathrm{p}=0.000$.

\section{Cross-sectional analysis}

The results of the cross-sectional analyses showed no significant association between objectively measured percentage of sedentary time and mental distress at baseline (table 2). There was a significant relationship between self-reported screen time and mental distress at baseline in the crude, partially and fully adjusted model. Objectively measured
Table 1 Frequencies and percentages of $\mathrm{T} 1$ subject characteristics

\begin{tabular}{|c|c|}
\hline & All $(n=686)$ \\
\hline \multicolumn{2}{|l|}{ Sex } \\
\hline Boys & $312(45.5 \%)$ \\
\hline \multicolumn{2}{|l|}{ Chronic pain $\mathrm{T} 1 \geq 3$ months } \\
\hline Yes & $165(24.1 \%)$ \\
\hline \multicolumn{2}{|l|}{ SES mother $\mathrm{T} 1$} \\
\hline High $\geq$ Higher education & $286(41.7 \%)$ \\
\hline \multicolumn{2}{|l|}{ SES father $\mathrm{T} 1$} \\
\hline High $\geq$ Higher education & $242(35.3 \%)$ \\
\hline \multicolumn{2}{|l|}{ Sleep onset latency (>30 min) T1 } \\
\hline Yes & $249(36.3 \%)$ \\
\hline \multicolumn{2}{|l|}{ Using the ActiGraph in the winter } \\
\hline $\mathrm{T} 1$ & $546(79.6 \%)$ \\
\hline \multicolumn{2}{|l|}{ HSCL-10 T1 } \\
\hline Over cut-off & $138(20.1 \%)$ \\
\hline \multicolumn{2}{|l|}{ HSCL-10 T2 } \\
\hline Over cut-off & $183(26.7 \%)$ \\
\hline \multicolumn{2}{|l|}{ MVPA (min/day) } \\
\hline Under $60 \mathrm{~min}$ & $594(86.6 \%)$ \\
\hline Age (years) & $16.25(0.94)$ \\
\hline Self-reported mean screen time per day ${ }^{\star}$ & $4.00(2.30)$ \\
\hline $\begin{array}{l}\text { Objectively measured sedentary time per } \\
\text { day* }\end{array}$ & $9.58(2.25)$ \\
\hline MVPA (minutes per day) & $44.66(18.06)$ \\
\hline BMI $\left(\mathrm{kg} / \mathrm{m}^{2}\right)$ & $22.27(3.95)$ \\
\hline Self-reported health (scale of $1-5$ ) & $3.94(0.83)$ \\
\hline Social network (scale of 1-4) & $3.30(0.48)$ \\
\hline
\end{tabular}

Time reported in hours per day.

BMI, body mass index; HSCL, Hopkins symptoms checklist; MVPA, Moderate to vigorous physical activity; SES, socioeconomic status; $\mathrm{T} 1$, baseline.

percentage of time spent in sedentary behaviour was not significantly related to mental distress at baseline.

The longitudinal relationship between percentage of sedentary behaviour and change in mental distress

Objectively assessed percentage of time in sedentary behaviour at baseline showed no significant relationship with change in mental distress between baseline and follow-up in neither crude, partly adjusted nor multiple adjusted analyses (table 3 ). Of the covariates, sex, mental distress at baseline, minutes in MVPA, sleep onset latency, chronic pain and screen time were significant predictors of mental distress at $\mathrm{T} 2$.

Self-reported screen time at baseline was significantly associated with increased mental distress after 2 years (table 4 ). Of the covariates, sex, mental distress at baseline, minutes in MVPA, sleep onset latency and chronic pain were significant predictors of mental distress at follow-up. 
Table 2 The cross-sectional association between baseline percentage of sedentary behaviour measured by accelerometer/ self-reported screen time and standardised HSCL-10 score at T1 investigated by multiple hierarchic linear regression

\section{Objectively measured percentage of sedentary}

behaviour*

Self-reported screen time†

\begin{tabular}{|c|c|c|c|c|c|c|c|c|}
\hline & \multirow[b]{2}{*}{ B } & \multicolumn{2}{|l|}{$95 \% \mathrm{Cl}$} & \multirow[b]{2}{*}{$P$ value } & \multirow[b]{2}{*}{ B } & \multicolumn{2}{|l|}{$95 \% \mathrm{Cl}$} & \multirow[b]{2}{*}{$P$ value } \\
\hline & & Lower & Upper & & & Lower & Upper & \\
\hline \multicolumn{9}{|l|}{ Adjusted model Block 1} \\
\hline Predictor variable & 0.013 & -0.001 & 0.027 & 0.068 & 0.052 & 0.020 & 0.085 & 0.002 \\
\hline \multicolumn{9}{|l|}{ Adjusted model Block 2} \\
\hline Predictor variable & 0.014 & $-3.521 \mathrm{E}-5$ & 0.027 & 0.051 & 0.078 & 0.046 & 0.110 & 0.000 \\
\hline Sex & -0.506 & -0.654 & -0.358 & 0.000 & -0.578 & -0.727 & -0.429 & 0.000 \\
\hline Age & 0.080 & 0.004 & 0.156 & 0.040 & 0.081 & 0.006 & 0.156 & 0.034 \\
\hline SES father & 0.011 & -0.166 & 0.188 & 0.902 & 0.028 & -0.145 & 0.202 & 0.748 \\
\hline SES mother & -0.008 & -0.180 & 0.163 & 0.923 & 0.014 & -0.156 & 0.183 & 0.872 \\
\hline Season & -0.053 & -0.284 & 0.177 & 0.648 & -0.070 & -0.299 & 0.158 & 0.546 \\
\hline \multicolumn{9}{|l|}{ Adjusted model Block 3} \\
\hline Predictor variable & 0.011 & -0.004 & 0.026 & 0.143 & 0.048 & 0.017 & 0.079 & 0.002 \\
\hline Sex & -0.472 & -0.612 & -0.332 & 0.000 & -0.472 & -0.612 & -0.332 & 0.000 \\
\hline Age & 0.023 & -0.048 & 0.093 & 0.526 & 0.023 & -0.048 & 0.093 & 0.526 \\
\hline SES father & 0.016 & -0.146 & 0.179 & 0.842 & 0.016 & -0.146 & 0.179 & 0.842 \\
\hline SES mother & 0.027 & -0.130 & 0.183 & 0.739 & 0.027 & -0.130 & 0.183 & 0.739 \\
\hline Season & -0.047 & -0.258 & 0.164 & 0.664 & -0.047 & -0.258 & 0.164 & 0.664 \\
\hline MVPA & 0.005 & 0.000 & 0.009 & 0.043 & 0.005 & 0.000 & 0.009 & 0.043 \\
\hline BMI & -0.009 & -0.027 & 0.008 & 0.294 & -0.009 & -0.027 & 0.008 & 0.294 \\
\hline Sleep onset latency & 0.427 & 0.284 & 0.571 & 0.000 & 0.427 & 0.284 & 0.571 & 0.000 \\
\hline Social network & -0.358 & -0.503 & -0.212 & 0.000 & -0.358 & -0.503 & -0.212 & 0.000 \\
\hline Self-reported health & -0.122 & -0.211 & -0.034 & 0.007 & -0.122 & -0.211 & -0.034 & 0.007 \\
\hline Chronic pain & 0.423 & 0.264 & 0.583 & 0.000 & 0.423 & 0.264 & 0.583 & 0.000 \\
\hline Control variableł & 0.048 & 0.017 & 0.079 & 0.002 & 0.011 & -0.004 & 0.026 & 0.143 \\
\hline
\end{tabular}

T1 (baseline) variables: age, SES mother/father (socioeconomic status $\geq$ higher education), season (winter), sleep onset latency $>30 \mathrm{~min}$, social network, self-reported health, chronic pain $>3$ months.

*Predictor variable, percentage of day spent in sedentary behavior per valid day.

†Predictor variable, screen time-time reported in hours per day.

$¥$ Screen time for when sedentary time is predictor, and sedentary time when screen time is predictor.

BMI, body mass index; HSCL, Hopkins Symptoms Checklist; MVPA, moderate to vigorous physical activity.

Interaction effects between percentage of sedentary time and self-reported screen time were investigated in the multiple adjusted model in separate analyses, but there were no significant effects. This was also true for the interaction effects independently between percentage of sedentary time/self-reported screen time and sex, mental health at baseline, MVPA, social network and BMI. Additional analyses investigating weekday and weekend screen time as separate predictors of mental distress showed the same result as mean weekday, and consequently, the results are not shown.

\section{Sensitivity analysis on original data}

Participants dropping out between T1 and T2 did not differ in terms of percentage of sedentary time, screen time, MVPA or symptoms of anxiety or depression. The results from the analyses on cases with complete data on the variables in question showed no significant association between percentage of sedentary time and change in mental distress, see table 5 . No significant association was also found between screen time and change in mental distress. The regression coefficients for percentage of sedentary behaviour per valid day were slightly lower, whereas the coefficients for screen time were slightly higher in Block 2 and 3 in the analyses of the non-imputed data, compared with the imputed data shown in tables 3 and 4 .

\section{DISCUSSION}

Screen time at baseline (in middle adolescence) was a significant predictor of increasing mental distress from middle to late adolescence, after adjusting for mental distress at baseline, whereas the association between objectively measured percentage of sedentary time at 
Table 3 The association between baseline percentage of sedentary time per valid day measured by accelerometer and standardised HSCL-10 score between baseline and T2, controlling for HSCL-10 at baseline, assessed by hierarchic linearly regression

\begin{tabular}{|c|c|c|c|c|c|c|}
\hline & \multirow[b]{2}{*}{ B } & \multicolumn{2}{|l|}{$95 \% \mathrm{Cl}$} & \multirow[b]{2}{*}{$P$ value } & \multirow[b]{2}{*}{$\mathbf{R}^{2}$} & \multirow[b]{2}{*}{ R change } \\
\hline & & Lower & Upper & & & \\
\hline Adjusted model Block 1 & & & & & 0.341 & 0.341 \\
\hline Sedentary time* & 0.004 & -0.007 & 0.016 & 0.460 & & \\
\hline HSCL-10 T1 & 1.096 & 0.980 & 1.211 & 0.000 & & \\
\hline Adjusted model Block 2 & & & & & 0.354 & 0.014 \\
\hline Sedentary time ${ }^{\star}$ & 0.005 & -0.007 & 0.016 & 0.399 & & \\
\hline HSCL-10 T1 & 1.047 & 0.927 & 1.167 & 0.000 & & \\
\hline Sex & -0.233 & -0.360 & -0.106 & 0.000 & & \\
\hline Age & -0.006 & -0.071 & 0.058 & 0.848 & & \\
\hline SES father & -0.016 & -0.163 & 0.132 & 0.835 & & \\
\hline SES mother & 0.044 & -0.098 & 0.187 & 0.541 & & \\
\hline Season & -0.009 & -0.198 & 0.180 & 0.925 & & \\
\hline Adjusted model Block 3 & & & & & 0.388 & 0.034 \\
\hline Sedentary time* & 0.009 & -0.004 & 0.023 & 0.189 & & \\
\hline HSCL-10 T1 & 0.870 & 0.732 & 1.008 & 0.000 & & \\
\hline Sex & -0.289 & -0.419 & -0.160 & 0.000 & & \\
\hline Age & -0.009 & -0.073 & 0.055 & 0.779 & & \\
\hline SES father & -0.024 & -0.170 & 0.122 & 0.747 & & \\
\hline SES mother & 0.046 & -0.095 & 0.186 & 0.524 & & \\
\hline Season & -0.017 & -0.206 & 0.171 & 0.856 & & \\
\hline MVPA & 0.005 & 0.000 & 0.009 & 0.032 & & \\
\hline BMI & -0.005 & -0.021 & 0.011 & 0.537 & & \\
\hline Sleep onset latency & 0.246 & 0.114 & 0.379 & 0.000 & & \\
\hline Social network & -0.121 & -0.257 & 0.016 & 0.084 & & \\
\hline Self-reported health & -0.010 & -0.092 & 0.071 & 0.807 & & \\
\hline Chronic pain & 0.198 & 0.050 & 0.347 & 0.009 & & \\
\hline Screen time & 0.038 & 0.010 & 0.066 & 0.008 & & \\
\hline
\end{tabular}

T1 (baseline) variables: age, SES mother/father (socioeconomic status $\geq$ higher education), season (winter), sleep onset latency $>30$ min, social network, self-reported health, chronic pain $>3$ months.

${ }^{*}$ Percentage of day spent in sedentary behaviour per valid day.

BMI, body mass index; HSCL, Hopkins Symptoms Checklist; MVPA, moderate to vigorous physical activity.

baseline and change in mental distress between middle to late adolescence was not significant. Similar associations were found in the cross-sectional analyses on baseline data, in which a significant relationship was found only between self-reported screen time and mental distress. This indicates that measurement methods may be of importance in the statistical relationship between sedentary behaviour and mental health, and that selfreported screen time may not be a fitting proxy for sedentary behaviour. Researchers should therefore thoroughly assess the validity and reliability of the chosen method of measurement in relation to the concept they wish to investigate.

The discrepancy in the results necessitates a discussion regarding the use of self-reported screen time measurements versus accelerometer measurements of sedentary behaviour. The mean levels of screen time per day (4hours) and sedentary time ( 9 hours) reported in the present study reveal a large difference, which nonetheless comply with mean levels shown in previous studies. ${ }^{46} 47$ Additionally, less than $15 \%$ of the adolescents were sufficiently active, $60 \mathrm{~min}$ of MVPA or more, ${ }^{48}$ a finding that is also in compliance with results of previous studies. ${ }^{40} 50$ Self-reported measurements are prone to report bias, and adolescents have been found to over-report time spent in MVPA $^{51}$ as well as to under-report minutes in sedentary behaviour ${ }^{52}$ compared with objective measurements. One study found that self-reported screen time correctly measured sedentary time in boys, ${ }^{53}$ whereas other studies conclude that self-reported screen time shows poor accuracy compared with objectively measured data. ${ }^{27}$ This current study may contribute to the evidence that 
Table 4 The association between self-reported screen time at baseline and standardised HSCL-10 score between baseline and follow-up, assessed by hierarchic linearly regression

\begin{tabular}{|c|c|c|c|c|c|c|}
\hline & & $95 \% \mathrm{Cl}$ & & & & \\
\hline & B & Lower & Upper & $P$ value & $\mathbf{R}^{2}$ & R change \\
\hline Adjusted model Block 1 & & & & & 0.344 & 0.344 \\
\hline Screen time* ${ }^{*}$ & 0.027 & 0.001 & 0.054 & 0.045 & & \\
\hline HSCL-10 T1 & 1.088 & 0.973 & 1.203 & 0.000 & & \\
\hline Adjusted model Block 2 & & & & & 0.362 & 0.018 \\
\hline Screen time ${ }^{*}$ & 0.042 & 0.014 & 0.069 & 0.003 & & \\
\hline HSCL-10 T1 & 1.024 & 0.903 & 1.144 & 0.000 & & \\
\hline Sex & -0.278 & -0.408 & -0.148 & 0.000 & & \\
\hline Age & -0.003 & -0.067 & 0.061 & 0.918 & & \\
\hline SES father & -0.008 & -0.154 & 0.137 & 0.911 & & \\
\hline SES mother & 0.056 & -0.086 & 0.198 & 0.438 & & \\
\hline Season & -0.018 & -0.206 & 0.170 & 0.852 & & \\
\hline Adjusted model Block 3 & & & & & 0.388 & 0.026 \\
\hline Screen time ${ }^{*}$ & 0.038 & 0.010 & 0.066 & 0.008 & & \\
\hline HSCL-10 T1 & 0.870 & 0.732 & 1.008 & 0.000 & & \\
\hline Sex & -0.289 & -0.419 & -0.160 & 0.000 & & \\
\hline Age & -0.009 & -0.073 & 0.055 & 0.779 & & \\
\hline SES father & -0.024 & -0.170 & 0.122 & 0.747 & & \\
\hline SES mother & 0.046 & -0.095 & 0.186 & 0.524 & & \\
\hline Season & -0.017 & -0.206 & 0.171 & 0.856 & & \\
\hline MVPA & 0.005 & 0.000 & 0.009 & 0.032 & & \\
\hline BMI & -0.005 & -0.021 & 0.011 & 0.537 & & \\
\hline Sleep onset latency & 0.246 & 0.114 & 0.379 & 0.000 & & \\
\hline Social network & -0.121 & -0.257 & 0.016 & 0.084 & & \\
\hline Self-reported health & -0.010 & -0.092 & 0.071 & 0.807 & & \\
\hline Chronic pain & 0.198 & 0.050 & 0.347 & 0.009 & & \\
\hline Sedentary time & 0.009 & -0.004 & 0.023 & 0.189 & & \\
\hline
\end{tabular}

T1 (baseline) variables: age, SES mother/father (socioeconomic status $\geq$ higher education), season (winter), sleep onset latency $>30$ min, social network, self-reported health, chronic pain $>3$ months, sedentary time-percentage of sedentary behaviour per valid day.

${ }^{*}$ Time reported in hours per day.

BMI, body mass index; HSCL, Hopkins Symptoms Checklist; MVPA, moderate to vigorous physical activity.

self-reported screen time is not a fitting proxy to sedentary time and that different types of measurements may give different results. This may have implications for further research on sedentary behaviour as researchers should thoroughly assess what type of measurement is suited to measure the behaviour. In addition, clinicians may consider the complexity of the self-reported estimation of sedentary behaviours, such as screen time, as it may be influenced by a number of psychological factors that may include depressive symptoms.

In general, studies of the association between sedentary behaviour and mental distress in adolescents have mainly used self-report measurements for sedentary behaviour, and the results from these studies commonly show sedentary behaviour to predict higher levels of mental distress. ${ }^{6}{ }^{19} 54-56$ A review found only evidence for a significant association between self-reported sedentary time and depression among adolescents, while anxiety and overall psychological distress was considered inconclusive. ${ }^{5}$ However, it is important to point out that the effect between screen time and mental health is generally small. ${ }^{525758}$ This is true also for the present study as supplementary analysis showed that screen time at baseline only accounts for $1.4 \%$ of the variance in mental distress 2years later, before adjustment of possible confounders. Orben and Przybylski ${ }^{25}$ have argued that the observed effect of screen time on well-being among adolescents is too small to justify any policy changes.

Our study is one of few who have examined the association between objectively measured sedentary behaviour and mental distress. Likewise, few studies of self-reported or objectively measured physical activity have applied longitudinal designs. The results of the few existing longitudinal studies tend to vary-although most have reported 
Table 5 The association between baseline sedentary time measured by accelerometer/self-reported screen time and change in mean score HSCL-10 between T1 and T2-complete cases, assessed by hierarchic linearly regression

\begin{tabular}{|c|c|c|c|c|c|c|}
\hline & \multicolumn{3}{|c|}{ Percentage of sedentary behaviour* } & \multicolumn{3}{|c|}{ Screen time } \\
\hline & B & $95 \% \mathrm{Cl}$ & $P$ value & B & $95 \% \mathrm{Cl}$ & P value \\
\hline \multicolumn{7}{|c|}{ Adjusted model Block $1 \ddagger$} \\
\hline \multicolumn{7}{|c|}{ Adjusted model Block $2 \S$} \\
\hline Predictor variable & 0.005 & $-0.008 / 0.019$ & 0.438 & 0.027 & $-0.013 / 0.067$ & 0.188 \\
\hline
\end{tabular}

${ }^{*}$ Percentage of day spent in sedentary behaviour.

†Time reported in hours per day.

łBlock 1: model adjusted by HSCL-10 (T1).

§Block 2: model adjusted by variables in Block 1; demography-related variables: sex, age, socioeconomic status father (>higher education T1), socioeconomic status mother (>higher educationT1), season (Winter T1), MVPA (T1), BMI (T1), sleep onset latency (>30 min T1), social network (T1) and screen time when sedentary time was predictor or sedentary time when screen time was predictor (T1).

IBlock 3: model adjusted by variables in Block 1, Block 2 and health-related variables: self-reported health (T1) and chronic pain (T1). BMI, body mass index; HSCL, Hopkins Symptoms Checklist; MVPA, moderate to vigorous physical activity; T1, baseline.

a significant, but small effect of self-reported sedentary behaviour (eg, see previous works 25759 ). Hamer et $a t^{28}$ compared the association between objectively assessed and self-reported sedentary behaviour with mental distress in adults in a cross-sectional study, and found that sedentary behaviour, independent of assessment method, was associated with adverse mental health. In contrast, a study by Hume $e t a l^{17}$ investigating the association between sedentary behaviour as measured by accelerometer, selfreported time spent watching TV and depressive symptoms in adolescent boys and girls found no significant association between sedentary behaviour or time spent watching TV and depressive symptoms in neither a crosssectional nor a longitudinal design. However, girls who were depressed at baseline spent significantly more time watching TV at follow-up. As studies on adults ${ }^{28} 60$ show a relationship between objectively measured sedentary behaviour and mental distress, as opposed to the current study and the study by Hume $e t a l,{ }^{17}$ it is reasonable to question whether sedentary behaviour may have different psychological impact on adults and adolescents. A study by Zahl et al found that objectively measured sedentary time among Norwegian children was not associated with depression 2years later, further pointing to the potential age-dependent effect of sedentary behaviour on mental distress.

It is possible that the association between sedentary behaviour and mental distress is more complex than what our study was able to investigate. We lack information regarding the context in which the adolescent is sedentary, for instance, if he/she is alone or with friends, playing online games with other players and so on. This social component of sedentary behaviour may impact the relationship with mental distress. Mentally active sedentary behaviour, such as office work or knitting/sewing, shows a potential positive effect on mental well-being in adults compared with passive sedentary behaviour. ${ }^{61}$ Hallgren $e t a l^{62}$ found a $5 \%$ decreased risk of major depressive disorder if $30 \mathrm{~min}$ of passive sedentary behaviour was replaced with equal amounts of mentally active sedentary behaviour. Future studies should aim to identify how specific modes of sedentary behaviour, such as time spent with friends, school classes or screen time, relates to mental distress. Studies that provide knowledge on adolescents' sedentary behaviour patterns and possible effect modifiers such as self-esteem and well-being are also required to fully understand the association between sedentary behaviour and mental distress.

\section{Methodological considerations}

The results are based on data from a relatively large sample with repeated measurements, making it possible to investigate the association between sedentary behaviour and mental distress over time. A major strength is that the study contains information on sedentary behaviour both from accelerometer data and from self-reported screen time. This enabled investigation of the effect of measurement method on the relationship to mental distress, as well as the validity of screen time as a proxy measure of sedentary behaviour. In addition, the study included adjustment for several potential confounders.

Despite these strengths, the results should be interpreted in light of certain methodological limitations. Even though objective measurements of physical activity and sedentary behaviour is considered more accurate and unbiased than self-report, there are still some validity issues that may have had an impact on the results. One issue is that accelerometers lack the ability to reliably register different types of activities such as rowing and cycling because of its static position on the hip. In addition, hip worn accelerometers have difficulty differentiating between postures, such as standing, sitting or 
lying down. ${ }^{63}$ In the current study, the inclinometer was not included because of the validity issues, but including posture in the analyses may have had a slight impact on the results, although it is unlikely to be a great source of bias. Reliably differentiating between sedentary time and non-wear time may also be challenging, and potentially lead to underestimation of sedentary time measured by accelerometer. ${ }^{64}$ Additionally, the study used data collected over a short period of wear time ( 7 days), and it is possible that these data are not representative of the participants' typical level of physical activity. Yet, research conducted on blinded and non-blinded participants show that the awareness of wearing an accelerometer has no effect on the pattern of physical activity in youth. ${ }^{65}$ Physical activity levels may vary by season, but almost all participants were measured during winter. Preliminary analyses also showed that there was no significant difference in levels of MVPA or sedentary behaviour for participants measured in winter or summer (results not shown). Another limitation of the study is that data on screen time and mental distress were collected through self-report. Self-reported data may be subject to response bias, in that behaviour or characteristics valued as positive may be over-reported, and negative characteristics under-reported. Furthermore, a relatively large amount of data was imputed, due to the high percentage of missing values. To prevent biassed estimations in multiple imputations, missing data must be missing at random. There is always a chance that the missing variables are not missing at random, as we do not know the reason for why the data are missing. However, the degree to which the participants with missing values differ from the participants with valid data may give us an indication for 'the missing pattern'. In the current study, the two groups of participants did not differ significantly from each other, and the results of the hierarchical regression analyses on the sample with complete data were in essence similar to the analyses of the imputed sample. This suggests a higher probability for missing at random and provides confidence that the imputation provided unbiased estimates, even with higher proportions of missing data. ${ }^{66}$ The single-item chronic pain assessment in this study was rather high, at $24.1 \%$. Nevertheless, similar results have been shown in other studies. ${ }^{67} 68$ The social network variable is not from a validated test battery, and as such, the validity of the measure is uncertain. This needs to be taken into account when interpreting the results. In addition, there may be other potential confounders that the study fail to control for, such as, the difference between passive and mentally active objectively measured sedentary behaviour, as research have shown that mentally active sedentary behaviour may have a positive impact on depression combined with light physical activity for adults. ${ }^{6162}$

Finally, the findings may not be generalisable to adolescents as a whole, because the participants in this study were collected from only two municipalities in the northern region of Norway. Nevertheless, the findings of percentage of sedentary time and minutes in MVPA resemble those from previous findings from national and European samples, which suggests that the participants are no more or less active or sedentary than other adolescents. Still, Northern Norway's long winter season and polar nights may have affected the results, and caution with generalising the findings is advised.

\section{CONCLUSION}

The study indicates a disparity in the results depending on the type of measurement method; self-reported screen time was associated with increased mental distress 2 years later, whereas objectively measured time in sedentary behaviour was not. This calls for a thorough assessment of the validity and reliability of the chosen method of measurement in relation to the concept one wishes to investigate.

\section{Author affiliations}

${ }^{1}$ Department of Psychology, UiT Arctic University of Norway, Tromso, Norway ${ }^{2}$ School of Sports Sciences, UiT Arctic University of Norway, Tromso, Norway ${ }^{3}$ Regional Centre for Child and Youth Mental Health and Child Welfare, UiT Arctic University of Norway, Tromso, Norway

${ }^{4}$ Work Research Institute, Oslo Metropolitan University, Oslo, Norway ${ }^{5}$ Chronic Diseases and Ageing, Nasjonalt Folkehelseinstitutt, Oslo, Norway ${ }^{6}$ Department of Community Medicine, UiT Arctic University of Norway, Tromso, Norway

${ }^{7}$ Department of Microbiology and Infection Control, Universitetssykehuset NordNorge, Tromso, Norway

${ }^{8}$ School of Psychiatry, University of New South Wales, Sydney, New South Wales, Australia

${ }^{9}$ Black Dog Institute, Randwick, New South Wales, Australia

Contributors Study concept and design: IMO, KR and BM. Analysing and interpreting of data: IMO, KR, BM and B-HH. Drafting of the manuscript: IMO. Critical revision of the manuscript for important intellectual content: IMO, KR, BM, B-HH, KRL, WN, CSN, A-SF and SR. Study supervision: KR. All authors read and approved the final manuscript.

Funding The Northern Norway Regional Health Authority (Norwegian: Helse Nord) funded the research when a layout of the project was approved by their committee December 2016. The Troms $\emptyset$ Study: Fit Futures is a collaborative study between the University Hospital of North Norway, UiT-The Arctic University of Norway and the Norwegian Institute of Public Health. ActiGraph instruments were funded by the Simon $\mathrm{F}$ Hartmann family fund.

\section{Competing interests None declared.}

Patient consent for publication Not required.

Ethics approval Norwegian Data Inspectorate and the Regional Committee for Medical and Health Research Ethics for Northern-Norway (REK-Nord) have approved Fit Futures - part of The Troms $\emptyset$ Study. The Troms $\emptyset$ Study collected the written informed consent from participants/guardians. The present project has received a specific approval from REK-Nord (Project number: 2016/987/REK nord).

Provenance and peer review Not commissioned; externally peer reviewed.

Data availability statement Data may be obtained from a third party and are not publicly available. The dataset supporting the articles findings is available through application directed to the Troms $\emptyset$ Study by following the steps presented on their online page: https://en.uit.no/forskning/forskningsgrupper/sub?p_document_id= 453582\&sub_id=71247.

Open access This is an open access article distributed in accordance with the Creative Commons Attribution Non Commercial (CC BY-NC 4.0) license, which permits others to distribute, remix, adapt, build upon this work non-commercially, and license their derivative works on different terms, provided the original work is properly cited, appropriate credit is given, any changes made indicated, and the use is non-commercial. See: http://creativecommons.org/licenses/by-nc/4.0/. 
ORCID iD

Ida Marie Opdal http://orcid.org/0000-0002-7092-9494

\section{REFERENCES}

1 Kessler RC, Bromet EJ. The epidemiology of depression across cultures. Annu Rev Public Health 2013;34:119-38.

2 Polanczyk GV, Salum GA, Sugaya LS, et al. Annual research review: a meta-analysis of the worldwide prevalence of mental disorders in children and adolescents. J Child Psychol Psychiatry 2015;56:345-65.

3 Ruiz JR, Ortega FB, Martínez-Gómez D, et al. Objectively measured physical activity and sedentary time in European adolescents: the Helena study. Am J Epidemiol 2011;174:173-84.

4 Johnson KE, Taliaferro LA. Relationships between physical activity and depressive symptoms among middle and older adolescents: a review of the research literature. J Spec Pediatr Nurs 2011;16:235-51.

5 Rodriguez-Ayllon M, Cadenas-Sánchez C, Estévez-López F, et al. Role of physical activity and sedentary behavior in the mental health of preschoolers, children and adolescents: a systematic review and meta-analysis. Sports Medicine 2019;49:1383-410.

6 Kremer P, Elshaug C, Leslie E, et al. Physical activity, leisure-time screen use and depression among children and young adolescents. J Sci Med Sport 2014;17:183-7.

7 Zahl T, Steinsbekk S, Wichstrøm L. Physical activity, sedentary behavior, and symptoms of major depression in middle childhood. Pediatrics 2017;139:e20161711.

8 Schuch FB, Vancampfort D, Firth J, et al. Physical activity and incident depression: a meta-analysis of prospective cohort studies. Am J Psychiatry 2018;175:631-48.

9 Vagetti GC, Barbosa Filho VC, Moreira NB, et al. Association between physical activity and quality of life in the elderly: a systematic review, 2000-2012. Rev. Bras. Psiquiatr. 2014;36:76-88.

10 Birkeland MS, Torsheim T, Wold B. A longitudinal study of the relationship between leisure-time physical activity and depressed mood among adolescents. Psychol Sport Exerc 2009;10:25-34.

11 Oweis P, Spinks W. Biopsychological, affective and cognitive responses to acute physical activity. J Sports Med Phys Fitness 2001;41:528-38.

12 Salmon P. Effects of physical exercise on anxiety, depression, and sensitivity to stress: a unifying theory. Clin Psychol Rev 2001;21:33-61.

13 Lubans D, Richards J, Hillman C, et al. Physical activity for cognitive and mental health in youth: a systematic review of mechanisms. Pediatrics 2016;138:e20161642.

14 Bailey AP, Hetrick SE, Rosenbaum S, et al. Treating depression with physical activity in adolescents and young adults: a systematic review and meta-analysis of randomised controlled trials. Psychol Med 2018;48:1068-83.

15 Toseeb U, Brage S, Corder K, et al. Exercise and depressive symptoms in adolescents: a longitudinal cohort study. JAMA Pediatr 2014;168:1093-100.

16 Rothon C, Edwards P, Bhui K, et al. Physical activity and depressive symptoms in adolescents: a prospective study. BMC Med 2010;8:32.

17 Hume C, Timperio A, Veitch J, et al. Physical activity, sedentary behavior, and depressive symptoms among adolescents. J Phys Act Health 2011;8:152-6.

18 Pearson N, Braithwaite RE, Biddle SJH, et al. Associations between sedentary behaviour and physical activity in children and adolescents: a meta-analysis. Obes Rev 2014;15:666-75.

19 Hoare E, Milton K, Foster C, et al. The associations between sedentary behaviour and mental health among adolescents: a systematic review. Int J Behav Nutr Phys Act 2016;13:108.

20 Arbour-Nicitopoulos KP, Faulkner GE, Irving HM. Multiple health-risk behaviour and psychological distress in adolescence. Journal of the Canadian Academy of Child Adolescent Psychiatry 2012;21:171.

21 Mathers M, Canterford L, Olds T, et al. Electronic media use and adolescent health and well-being: cross-sectional community study. Acad Pediatr 2009:9:307-14.

22 Bickham DS, Hswen Y, Rich M. Media use and depression: exposure, household rules, and symptoms among young adolescents in the USA. Int J Public Health 2015;60:147-55.

23 Primack BA, Swanier B, Georgiopoulos AM, et al. Association between media use in adolescence and depression in young adulthood. Arch Gen Psychiatry 2009;66:181-8.

24 Stiglic N, Viner RM. Effects of screentime on the health and wellbeing of children and adolescents: a systematic review of reviews. BMJ Open 2019;9:e023191.
25 Orben A, Przybylski AK. The association between adolescent wellbeing and digital technology use. Nat Hum Behav 2019;3:173-82.

26 Brunborg GS, Burdzovic Andreas J. Increase in time spent on social media is associated with modest increase in depression, conduct problems, and episodic heavy drinking. J Adolesc 2019;74:201-9.

27 Chastin SFM, Dontje ML, Skelton DA, et al. Systematic comparative validation of self-report measures of sedentary time against an objective measure of postural sitting (activPAL). Int J Behav Nutr Phys Act 2018;15:21

28 Hamer M, Coombs N, Stamatakis E. Associations between objectively assessed and self-reported sedentary time with mental health in adults: an analysis of data from the health survey for England. BMJ Open 2014;4:e004580.

29 Christoffersen T, Winther A, Nilsen OA, et al. Does the frequency and intensity of physical activity in adolescence have an impact on bone? The Tromsø study, fit futures. BMC Sports Sci Med Rehabil 2015;7:26.

30 Opdal IM, Morseth $\mathrm{B}$, Handegård $\mathrm{BH}$, et al. Change in physical activity is not associated with change in mental distress among adolescents: the Tromsø study: fit futures. BMC Public Health 2019;19:916.

31 Migueles $\mathrm{JH}$, Cadenas-Sanchez C, Ekelund U, et al. Accelerometer data collection and processing criteria to assess physical activity and other outcomes: a systematic review and practical considerations. Sports Med 2017;47:1821-45.

32 Freedson PS, Melanson E, Sirard J. Calibration of the computer science and applications, Inc. accelerometer. Med Sci Sports Exerc 1998:30:777-81.

33 Haavet OR, Sirpal MK, Haugen W, et al. Diagnosis of depressed young people in primary health care-a validation of HSCL-10. Fam Pract 2011;28:233-7.

34 Strand BH, Dalgard OS, Tambs K, et al. Measuring the mental health status of the Norwegian population: a comparison of the instruments SCL-25, SCL-10, SCL-5 and MHI-5 (SF-36). Nord J Psychiatry 2003;57:113-8.

35 Sandanger I, Moum T, Ingebrigtsen G, et al. Concordance between symptom screening and diagnostic procedure: the Hopkins symptom Checklist-25 and the composite international diagnostic interview I. Soc Psychiatry Psychiatr Epidemiol 1998;33:345-54.

36 Gispert R, Rajmil L, Schiaffino A, et al. Sociodemographic and health-related correlates of psychiatric distress in a general population. Soc Psychiatry Psychiatr Epidemiol 2003;38:677-83.

37 Caspersen CJ, Pereira MA, Curran KM. Changes in physical activity patterns in the United States, by sex and cross-sectional age. Med Sci Sports Exerc 2000;32:1601-9.

38 Strine TW, Okoro CA, Chapman DP, et al. Health-related quality of life and health risk behaviors among smokers. Am J Prev Med 2005;28:182-7.

39 Wilson AC, Palermo TM. Physical activity and function in adolescents with chronic pain: a controlled study using actigraphy. J Pain 2012;13:121-30.

40 Janssen I, Katzmarzyk PT, Boyce WF, et al. Overweight and obesity in Canadian adolescents and their associations with dietary habits and physical activity patterns. J Adolesc Health 2004;35:360-7.

41 Strine TW, Chapman DP. Associations of frequent sleep insufficiency with health-related quality of life and health behaviors. Sleep Med 2005;6:23-7.

42 de Wit LM, Fokkema M, van Straten A, et al. Depressive and anxiety disorders and the association with obesity, physical, and social activities. Depress Anxiety 2010;27:1057-65.

43 Lichstein KL, Durrence HH, Taylor DJ, et al. Quantitative criteria for insomnia. Behav Res Ther 2003;41:427-45.

44 IBMCorp. Ibm SPSS statistics for windows, version 25.0. Armonk, NY: IBM Corp, 2017.

45 World Health Organization. Global recommendations on physical activity for health, 2010. Available: https://apps.who.int/iris/bitstream/ handle/10665/44399/9789241599979_eng.pdf;jsessionid=952EF2FE 7000ECC64D066DFA28FBD1D4?sequence=1

46 Arundell L, Salmon J, Koorts H, et al. Exploring when and how adolescents sit: cross-sectional analysis of activPAL-measured patterns of daily sitting time, bouts and breaks. BMC Public Health 2019;19:653

47 Bucksch J, Sigmundova D, Hamrik Z, et al. International trends in adolescent Screen-Time behaviors from 2002 to 2010. J Adolesc Health 2016:58:417-25.

48 World Health Organisation. (c) World Health organization 2010, 2010. Available: https://www.who.int/dietphysicalactivity/physical-activityrecommendations-18-64years.pdf?ua $=1$

49 McMahon EM, Corcoran P, O'Regan G, et al. Physical activity in European adolescents and associations with anxiety, depression and well-being. Eur Child Adolesc Psychiatry 2017;26:111-22. 
50 Cooper AR, Goodman A, Page AS, et al. Objectively measured physical activity and sedentary time in youth: the International children's accelerometry database (ICAD). Int J Behav Nutr Phys Act 2015;12:113.

51 Slootmaker SM, Schuit AJ, Chinapaw MJ, et al. Disagreement in physical activity assessed by accelerometer and self-report in subgroups of age, gender, education and weight status. Int J Behav Nutr Phys Act 2009;6:17.

52 Affuso O, Stevens J, Catellier D, et al. Validity of self-reported leisuretime sedentary behavior in adolescents. J Negat Results Biomed 2011;10:2.

53 Rey-López JP, Ruiz JR, Ortega FB, et al. Reliability and validity of a screen time-based sedentary behaviour questionnaire for adolescents: the Helena study. Eur J Public Health 2012;22:373-7.

54 Maras D, Flament MF, Murray M, et al. Screen time is associated with depression and anxiety in Canadian youth. Prev Med 2015;73:133-8.

55 Trinh L, Wong B, Faulkner GE. The independent and interactive associations of screen time and physical activity on mental health, school connectedness and academic achievement among a population-based sample of youth. J Can Acad Child Adolesc Psychiatry 2015;24:17-24.

56 Stanczykiewicz B, Banik A, Knoll N, et al. Sedentary behaviors and anxiety among children, adolescents and adults: a systematic review and meta-analysis. BMC Public Health 2019;19:459.

57 Sund AM, Larsson B, Wichstrøm L. Role of physical and sedentary activities in the development of depressive symptoms in early adolescence. Soc Psychiatry Psychiatr Epidemiol 2011;46:431-41.

58 Biddle SJH, Asare M. Physical activity and mental health in children and adolescents: a review of reviews. $\mathrm{Br} J$ Sports Med 2011;45:886-95.
59 Romer D, Bagdasarov Z, More E. Older versus newer media and the well-being of United States youth: results from a national longitudinal panel. J Adolesc Health 2013;52:613-9.

60 Vallance JK, Winkler EAH, Gardiner PA, et al. Associations of objectively-assessed physical activity and sedentary time with depression: NHANES (2005-2006). Prev Med 2011;53:284-8.

61 Hallgren M, Owen N, Stubbs B, et al. Passive and mentally-active sedentary behaviors and incident major depressive disorder: a 13year cohort study. J Affect Disord 2018;241:579-85.

62 Hallgren M, Nguyen T-T-D, Owen N, et al. Cross-sectional and prospective relationships of passive and mentally active sedentary behaviours and physical activity with depression. Br J Psychiatry 2019:1-7.

63 Kozey-Keadle S, Libertine A, Lyden K, et al. Validation of wearable monitors for assessing sedentary behavior. Med Sci Sports Exerc 2011;43:1561-7.

64 Oliver M, Badland HM, Schofield GM, et al. Identification of accelerometer nonwear time and sedentary behavior. Res $Q$ Exerc Sport 2011;82:779-83

65 Vanhelst J, Béghin L, Drumez E, et al. Awareness of wearing an accelerometer does not affect physical activity in youth. BMC Med Res Methodol 2017;17:99.

66 Lee JH, Huber Jr J. Multiple imputation with large proportions of missing data: how much is too much? United Kingdom Stata users group meetings 2011. Stata Users Group, 2011.

67 Sundblad GMB, Saartok T, Engström L-MT, et al. Prevalence and co-occurrence of self-rated pain and perceived health in schoolchildren: age and gender differences. European Journal of Pain 2007;11:171-80.

68 Perquin CW, Hazebroek-Kampschreur AAJM, Hunfeld JAM, et al Pain in children and adolescents: a common experience. Pain 2000;87:51-8. 